\title{
Extreme Heat Awareness and Protective Behaviors in New York City
}

\author{
Kathryn Lane, Katherine Wheeler, Kizzy Charles-Guzman, \\ Munerah Ahmed, Micheline Blum, Katherine Gregory, \\ Nathan Graber, Nancy Clark, and Thomas Matte
}

\begin{abstract}
Heat waves can be lethal and routinely prompt public warnings about the dangers of heat. With climate change, extreme heat events will become more frequent and intense. However, little is known about public awareness of heat warnings or behaviors during hot weather. Awareness of heat warnings, prevention behaviors, and air conditioning (AC) prevalence and use in New York City were assessed using quantitative and qualitative methods. A random sample telephone survey was conducted in September 2011 among 719 adults and follow-up focus groups were held in winter 2012 among seniors and potential senior caregivers. During summer 2011, $79 \%$ of adults heard or saw a heat warning. Of the $24 \%$ who were seniors or in fair or poor health, $34 \%$ did not own AC or never/rarely used it on hot days. Of this subgroup, $30 \%$ were unaware of warnings, and $49 \%$ stay home during hot weather. Reasons for not using AC during hot weather include disliking AC (29\%), not feeling hot $(19 \%)$, and a preference for fans $(18 \%)$. Seniors in the focus groups did not perceive themselves to be at risk, and often did not identify $A C$ as an important health protection strategy. While heat warnings are received by most New Yorkers, AC cost, risk perception problems, and a preference for staying home leave many at risk during heat waves. Improving AC access and risk communications will help better protect the most vulnerable during heat waves.
\end{abstract}

KEYWORDS Climate change, Heat-health behaviors, Heat waves, Risk perception, Climate adaptation, Air conditioning

\section{BACKGROUND}

Heat waves kill more Americans, on average, each year than any other extreme weather event, and ambient heat causes more deaths than any other natural hazard, with the exception of cold weather. ${ }^{1,2}$ Impacts are generally more concentrated in urban areas like New York City (NYC) due to the density of populations at risk, and may be exacerbated by characteristics of the landscape that intensify heat. ${ }^{3}$ In the summer of 2006 , there were 46 heat stroke deaths in NYC associated with two July heat waves. Among cases where air conditioning (AC) status was ascertained, the vast majority did not have a working AC. ${ }^{4}$ In recent years, more than $80 \%$ of decedents have been exposed at home. ${ }^{5}$

Lane, Charles-Guzman, Ahmed, Clark, and Matte are with the Division of Environmental Health, New York City Department of Health and Mental Hygiene, New York, NY, USA; Wheeler and Graber were with the Division of Environmental Health, New York City Department of Health and Mental Hygiene, New York, NY, USA at the time of the study; Blum is with the Baruch College Survey Research, School of Public Affairs, Baruch College, City University of New York, New York, NY, USA; Gregory was in the Division of External Affairs, New York City Department of Health and Mental Hygiene, New York, NY, USA.

Correspondence: Kathryn Lane, Division of Environmental Health, New York City Department of Health and Mental Hygiene, New York, NY, USA. (E-mail: klane1@health.nyc.gov) 
Furthermore, the NYC Panel on Climate Change projects that summers will be hotter and the number and intensity of heat waves will continue to increase. ${ }^{6}$ Heat illness prevention efforts in NYC have focused on improving access to and promotion of the use of AC, the most effective intervention supported by the literature. ${ }^{4,7-11}$

Public messaging and the activation of emergency response plans are triggered by heat advisories, watches, and warnings released by the National Weather Service when the maximum heat index is forecast to reach $100^{\circ} \mathrm{F}$ for any period of time, or $95^{\circ} \mathrm{F}$ for at least two consecutive days.

During heat emergencies, NYC's Office of Emergency Management coordinates a multi-agency response that incorporates widely recommended best practices for protecting health during extreme heat events. ${ }^{12}$ The response includes extensive risk communication and opening hundreds of "cooling centers," which are air-conditioned public spaces, such as senior centers, public housing community rooms, and libraries that are open for extended hours and promoted as places where the public can seek relief from the heat. Public messaging is disseminated through press releases, heat safety brochures, email alerts to health care and social service providers, and emails and text alerts for members of the general public who sign up for emergency notifications. At-risk individuals are advised to visit a cooling center or use AC set at $78^{\circ} \mathrm{F}$ or "low cool" to lessen strain on the energy grid while providing an environment that is protective of health. Information is provided to organizations serving populations with special health needs so they can help vulnerable clients stay cool. Heat safety brochures and other materials are distributed year-round.

Despite these extensive heat-illness prevention efforts, little is known about whether at-risk populations and their social contacts are aware of heat warnings or take effective steps to protect their health during periods of extreme heat. Some evidence from other localities indicates that vulnerable groups may not be receiving necessary heat information, and that awareness of extreme heat warnings is not always coupled with an understanding of heat-health risk factors and behavior change. ${ }^{13}$

To inform improvements in heat risk communications and other public health measures, we conducted quantitative and qualitative studies about heat-health awareness and behaviors. First, a telephone survey of a probability sample of NYC adults was administered to examine AC access, heat-illness prevention behaviors, and awareness of heat-health warnings. Next, we convened focus groups to elicit qualitative information about heat-health knowledge and behaviors among seniors, who are among the most vulnerable to heat illness and death, and potential senior caregivers.

\section{METHODS}

\section{Survey}

In collaboration with Baruch College Survey Research, DOHMH developed a 15question telephone survey, which was conducted from 20 to 25 September 2011 in English or Spanish among 719 adults aged 18+ living in NYC. The survey followed a severe July 2011 heat wave where heat indices reached $115^{\circ} \mathrm{F}$.

Respondents were selected through a combination of landline and cell phone random digit dialing. The landline sample was based on a random digit dial design, and respondents were selected randomly within the household. Cell phone users were sampled using a mobile number database that contains all possible numbers in 100 blocks dedicated to wireless service and 100 blocks providing shared services but with no directory-listed telephone.

Data were weighted to 2009 US Census American Community Survey age, sex, race, Hispanic ethnicity, and borough data for NYC adults. The estimated margin of 
error was \pm 3.7 percentage points for the full sample. Analyses were conducted using SUDAAN 10.0.1 software. $T$ tests were used to determine whether prevalence differences between groups were statistically significant.

The 15 questions were close-ended and, when appropriate, respondents were asked to select the "most important" response from a list of options. Adults with high heat-health risk were defined as those who were either aged $\geq 65$ years or were younger adults reporting "poor" or "fair" health. Self-reported poor or fair health has been shown to consistently predict mortality and, among respondents to the NYC Community Health Survey, has been associated with conditions that increase susceptibility to heat illness, including diabetes, obesity, and high blood pressure $\left[{ }^{14,15}\right]$. Adults who reported having no AC, or using AC either "never" or "less than half the time" when they were at home during very hot weather were defined as having a high heat-exposure risk. A "most vulnerable" group was defined as adults with high heat-health risk and high heat-exposure risk.

\section{Focus Groups}

In winter 2012, DOHMH, in collaboration with Global Strategy Group, a research and strategic communications firm, convened focus groups among (1) seniors aged $\geq 65$ and (2) younger adults aged 18-64 who reported having a senior friend, relative, or neighbor living in NYC ("i.e., potential helpers" or caregivers). Each type of group was conducted in English and Spanish. The four groups had a total of 38 participants, with 9-10 participants per group. Each discussion lasted approximately 1.5 h. The groups were audiotaped, transcribed, and evaluated qualitatively.

Participants were selected to represent a mix of race/ethnicities and genders. Participants in the "potential helpers" groups were required to have at least one senior in their social network, and at least half were recruited from zip codes that fall within District Public Health Offices' (DPHO) catchment areas, which are lower-income neighborhoods in Brooklyn, the Bronx, and Manhattan that DOHMH has identified as having elevated rates of disease. ${ }^{16}$ In the seniors groups, participants aged $\geq 65$ were required to $(1)$ live in a DPHO catchment area or have a household income that was no more than $200 \%$ of the Federal Poverty Level and (2) not have a functioning AC in their home, or report using AC "never" or "less than half the time" when they were at home during very hot weather. Participants received a cash incentive to compensate for their time.

Senior focus group discussions covered knowledge of heat-health risks, the rationales for heat-health behaviors during hot weather, and barriers or enablers to protection. Participants were also asked to respond to heat-health education materials published by NYC and other municipal agencies, as well as to local TV news clips about extreme heat from summer 2011. DOHMH reviewed online television clips from English and Spanish news outlets and qualitatively selected relatively good examples (i.e., that conveyed some information about health risks and protective actions) for viewing. The "potential helpers" discussions covered similar topics, and also focused on attitudes and behaviors related to helping others during heat waves. The survey and focus groups were reviewed by the NYC DOHMH Institutional Review Board and were determined to be exempt research.

\section{RESULTS}

\section{Survey}

The survey cooperation rate (defined according to the American Association for Public Opinion Research Cooperation Rate 3 criteria) was $58 \% .{ }^{17}$ The majority of 
interviews were conducted on a landline (81\% landlines vs. $19 \%$ cell phones) and $7 \%$ of interviews were conducted in Spanish.

Demographic characteristics corresponded to characteristics in 2010 Census data for adult New Yorkers, although fewer Asians were sampled in our survey ( 9 vs. $13 \%$ in the 2010 census, $p=<0.001) .{ }^{18}$ The high heat-health risk group-those who reported either having poor or fair general health status or were aged $\geq 65$-comprised $24 \%$ of respondents. Altogether, $8 \%$ of adults had high heat-health risk and high heat-exposure "most vulnerable" risk (Table 1), which translates to approximately 545,000 (438,000676,000) New Yorkers.

TABLE 1 Characteristics of survey respondents

\begin{tabular}{|c|c|c|c|}
\hline & & Unweighted $(N)$ & Weighted (\%) \\
\hline Total & & 719 & 100 \\
\hline Sex & Male & 266 & 46 \\
\hline \multirow[t]{5}{*}{ Age } & $18-29$ & 139 & 23 \\
\hline & $30-49$ & 214 & 39 \\
\hline & $50-64$ & 169 & 22 \\
\hline & $65+$ & 186 & 16 \\
\hline & Missing/refused & 11 & \\
\hline \multirow[t]{6}{*}{ Race/ethnicity } & White non-Hispanic & 318 & 39 \\
\hline & Black non-Hispanic & 142 & 21 \\
\hline & Hispanic & 155 & 26 \\
\hline & Asian/PI non-Hispanic & 48 & 9 \\
\hline & Other non-Hispanic & 30 & 4 \\
\hline & Missing/refused & 26 & \\
\hline \multirow[t]{5}{*}{ Household income } & $<\$ 30,000$ & 191 & 33 \\
\hline & $\$ 30,000$ to $<\$ 50,000$ & 114 & 22 \\
\hline & $\$ 50,000$ to $<\$ 100,000$ & 140 & 25 \\
\hline & $\geq 100,000$ & 103 & 20 \\
\hline & Missing/refused & 171 & \\
\hline Aware of heat warning & & 552 & 79 \\
\hline \multirow[t]{3}{*}{ General health status } & Excellent/very good/good & 588 & 85 \\
\hline & Fair/poor & 125 & 15 \\
\hline & Missing/refused & 6 & \\
\hline Health risk status & High (age $65+$ or fair/poor health) & 243 & 24 \\
\hline \multirow[t]{4}{*}{ AC status } & No functioning AC & 82 & 11 \\
\hline & Used never or $<$ half time & 104 & 14 \\
\hline & Used half the time or more & 529 & 75 \\
\hline & Missing/refused & 4 & \\
\hline Exposure risk status & High & 186 & 25 \\
\hline Most vulnerable & High health and exposure risk & 86 & 8 \\
\hline
\end{tabular}

Missing data not included in percentages. Percentages may not sum to $100 \%$ due to rounding 
Respondents in this most vulnerable group had significantly lower household incomes compared to those with lower vulnerability, with $82 \%$ reporting a household income $<\$ 30,000(p=<0.001)$. A higher percentage of people in the most vulnerable group lived in the Bronx (26 vs. $15 \%$ of the total sample, $p=0.035$ ). Those who had incomes $<\$ 30,000$ or were aged $\geq 65$ were more likely to not own or use AC during very hot weather (Table 2).

In 2011, $11 \%$ did not have AC, and about $15 \%$ of adults who owned AC used it "less than half the time" or "never" during very hot weather. The most frequently cited barriers to AC ownership among those at high risk of heat illness were cost (54\%), the perception that AC was not needed (28\%), and a dislike of AC (16\%). A dislike of AC was a frequently cited reason (29\%) among those who do not use AC during very hot weather; another $18 \%$ volunteered that they prefer to use a fan instead (Table 3).

Among the most vulnerable group (high heat-health risk and high heat-exposure risk), $49 \%$ reported staying home during very hot weather even if they could not stay cool there (Table 3). A follow-up question asked those who stayed home to specify the most important reason and $72 \%$ responded that they preferred to stay home. In addition, $11 \%$ of those who stay home reported feeling unsafe leaving home and $7 \%$ cited health concerns.

Among the entire sample, $79 \%$ recalled that they heard or saw a heat warning during summer 2011. Those in the most vulnerable category were somewhat less likely to recall that they heard or seen a heat warning than those who are less vulnerable (70 vs. $80 \%(p=0.06)$; Table 4$)$. Among the most vulnerable who heard a warning, about the same proportion stayed home $(50 \%)$ as those who left home $(49 \%)$. Most respondents $(75 \%)$ received their heat information from TV; while about $82 \%$ of those in the vulnerable group received information about dangerous heat from TV and $13 \%$ from radio. During very hot weather, $54 \%$ of all respondents reported checking on a family member, friend, or neighbor.

TABLE 2 Frequency of high heat-exposure ${ }^{a}$ risk by demographic characteristics among survey respondents

High-exposure risk weighted \% $(95 \% \mathrm{Cl})$

\begin{tabular}{lll}
\hline Total (unweighted, $n=186)$ & & $25(22,28)$ \\
Sex & Male & $27(22,33)$ \\
& Female & $22(19,27)$ \\
Age $^{\mathrm{b}}$ & $18-29$ & $22(15,30)$ \\
& $30-49$ & $16(12,22)$ \\
& $50-64$ & $31(25,39)$ \\
& $65+$ & $40(33,47)$ \\
Household income $^{\mathrm{b}}$ & $<\$ 30,000$ & $42(34,49)$ \\
& $\geq \$ 30,000$ & $18(14,23)$ \\
Health risk status $^{\mathrm{b}}$ & High & $34(28,41)$ \\
& Low & $22(18,26)$ \\
\hline
\end{tabular}

a Respondents reported never having AC, or using AC either "never" or "less than half the time"

${ }^{\mathrm{b}}$ Proportion with high exposure significantly different among subgroups $(p<0.05)$ 
TABLE 3 Reasons for not having air conditioning (AC), AC use patterns, and AC-seeking behavior during hot weather among subset of most vulnerable ${ }^{\mathrm{a}}$ (unweighted, $n=86$ )

Weighted \% $(95 \% \mathrm{Cl})$

No $\mathrm{AC}^{\mathrm{b}}$ (unweighted, $n=40$ )

Can't afford AC

$54(37,69)$

Don't need AC

$28(16,45)$

Don't like AC

$16(8,30)$

Building wiring not equipped

$2(0,12)$

Never/infrequent AC use ${ }^{\mathrm{b}}$ (unweighted, $n=36$ )

Don't like AC

$29(16,46)$

Did not feel hot

$19(9,36)$

Want to conserve energy

$18(8,36)$

Prefer fan (volunteered only)

$18(8,36)$

Concerned about electric bill

$12(5,26)$

Went somewhere else to get cool

$4(1,24)$

Behavior during very hot weather (unweighted, $n=84$ )

Stay home even though you're hot

$49(38,60)$

Other ${ }^{\text {b }}$

$26(18,37)$

Community center, library, or other public place ${ }^{b}$

$10(5,20)$

Place of business ${ }^{b}$

$8(4,17)$

Someone else's home ${ }^{b}$

$6(2,16)$

Reasons for staying home ${ }^{b}$ (unweighted, $n=41$ )

Prefer to stay home

$72(56,83)$

Don't feel safe leaving home

$11(5,25)$

Health makes it hard to leave home

$7(2,19)$

Don't know where to go

$6(2,22)$

Don't want to leave a pet

$4(1,16)$

Don't have transport

0

a Survey respondents who reported not having AC, or using AC either "never" or "less than half the time" and were either aged $\geq 65$ years or reported "poor" or "fair" health status

${ }^{b}$ Estimate has a relative standard error (a measure of precision) above $30 \%$ or sample size $\leq 50$, making the estimate potentially unreliable. Estimate should be interpreted with caution

\section{Focus Groups}

Approximately two thirds of senior participants rated their health as good or very good. Potential caregivers reported numerous types of relationships with seniors, including friend, grandparent, and parent. Interactions with seniors included social visits, running errands, and meal preparation. Several themes emerged during the groups, which are summarized below.

Heat-Health Awareness and Risk Perception Gaps in risk perception were evident among both caregivers and seniors. Although most seniors knew heat could be dangerous to health and understood age is a risk factor for heat illness, they did not believe that heat was a major risk to their own health (i.e., it was dangerous to other seniors): "I am not personally afraid of heat." Some seniors were aware that chronic health conditions like heart disease and obesity can increase heat vulnerability while others were surprised that some medications can impair thermoregulation. While both groups accurately described 
TABLE 4 Heat warning awareness by demographic characteristics among survey respondents

\begin{tabular}{|c|c|c|}
\hline \multicolumn{2}{|l|}{ Characteristic } & \multirow{2}{*}{$\frac{\text { Weighted \% aware of warning }(95 \% \mathrm{Cl})}{79(76,82)}$} \\
\hline Total (unweighted, $n$ & & \\
\hline \multirow[t]{2}{*}{ Sex } & Male & $76(71,81)$ \\
\hline & Female & $82(78,85)$ \\
\hline \multirow[t]{4}{*}{$\operatorname{Age}^{\mathrm{a}}$} & $18-29$ & $76(68,82)$ \\
\hline & $30-49$ & $80(74,85)$ \\
\hline & $50-64$ & $85(79,90)$ \\
\hline & $65+$ & $75(67,81)$ \\
\hline \multirow[t]{2}{*}{ Household income } & $<\$ 30,000$ & $82(75,87)$ \\
\hline & $\geq \$ 30,000$ & $82(77,86)$ \\
\hline \multirow[t]{2}{*}{ Health risk ${ }^{b}$} & High & $79(73,84)$ \\
\hline & Low & $79(75,83)$ \\
\hline \multirow[t]{2}{*}{ Exposure risk $^{c}$} & High & $77(69,83)$ \\
\hline & Low & $80(76,84)$ \\
\hline \multirow[t]{2}{*}{ Vulnerability status $^{d}$} & High & $70(58,79)$ \\
\hline & Low & $80(77,83)$ \\
\hline
\end{tabular}

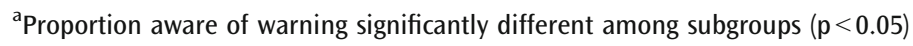

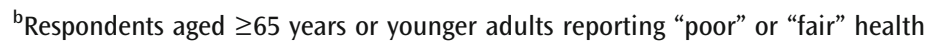

'Respondents who reported not having AC, or using AC either "never" or "less than half the time" during very hot weather

${ }^{d}$ Respondents who are aged $\geq 65$ years or younger adults reporting "poor" or "fair" health AND reported not having AC, or using AC either "never" or "less than half the time" during very hot weather

extreme heat conditions as including high temperatures and humidity, caregivers associated heat-related illness with outdoor physical activities and had not considered poorly cooled indoor environments as health risks for vulnerable people.

Behaviors During Hot Weather Although most seniors (79\%) reported having $\mathrm{AC}$, the majority reported using AC either never or less than half the time during hot weather. Some noted that they typically do not use AC but will use it when they are uncomfortable. Reasons for not using AC included not liking the feel, the cost of electricity, and energy conservation. Several seniors noted that they preferred using a fan over AC. Some believed AC could exacerbate health problems like arthritis and asthma. Seniors also reported that individuals may not actually feel hot during extreme heat, while others said they were "used" to the heat or that it was not as hot in NYC as in tropical locations: "I think it's only three months of summer here in New York, so three or four days it's really hot. Nothing happens."

Even those who were not regularly using AC often took some protective steps during hot weather such as drinking water, wearing light clothing, and going to a cooler place. Many fan users reported incorrectly using fans in rooms with closed windows during hot weather. Some thought that advice to use fans with open windows was not correct.

The majority of seniors were aware of cooling centers. Some had visited cooling centers and reported receiving heat-health information and having positive experiences. 
Others reported that they, or other seniors in their lives, did not visit cooling centers because of a preference to stay at home, a lack of interest in socializing, or not wanting to be around "old people." Many participants in all groups reported that they check on vulnerable contacts or knew of outreach from service organizations to homebound or vulnerable people during heat events.

Responses to Heat-Health Messages in News Media Participants did not identify $\mathrm{AC}$ use as an important preventive measure after viewing local news clips about extreme heat. Participants also noticed a discrepancy between TV voiceovers and images, which tended to depict children and younger adults in outdoor environments, rather than seniors and potentially dangerous indoor locations. Local TV health/ medical correspondents and meteorologists, doctors, and the health department were considered trustworthy sources of heat-health information.

\section{DISCUSSION}

Penetration of heat warnings was generally high among New Yorkers, however, awareness among the "most vulnerable" group was lower. Furthermore, nearly half of the most vulnerable group reported staying home during hot weather, where indoor temperature can be higher than outdoors during hot weather in the absence of AC. ${ }^{19}$ Stay-at-home behavior among this group was not associated with warning awareness, suggesting that messages in the warnings are not resonating with this group and/or that there are other important barriers to protective action. Reasons for not owning or using AC during very hot weather included cost, as well as a dislike of AC or a feeling that it was not needed, indicating that gaps in personal risk perception may also be an obstacle to heat-health protection. The focus groups also suggested a gap between risk awareness and personal risk perception.

Misconceptions about the benefits of electric fans may also impact risk perception. Retrospective observational studies in the USA have found no protective effect of electric fans during extreme heat. ${ }^{7,8,20,21}$ Researchers have theorized that circulation of ambient air that is cooler than normal body temperature is necessary for fans to provide effective cooling through convection. ${ }^{22}$ However, nearly $20 \%$ of survey respondents with a health risk factor who do not have AC or never/rarely used AC during very hot weather reported that the main reason was a preference for using a fan instead. Senior focus group participants expressed a similar preference for fan use during hot weather; many reported using fans incorrectly during hot weather.

Vulnerable people may have other reasons for staying home besides risk perception. While only a minority of NYC respondents cited health and safety concerns as reasons for staying home during hot weather, fears of crime or feeling that a neighborhood was unsafe were also reasons why residents stayed in unsafe home environments during the deadly 1995 Chicago heat wave. ${ }^{23}$ Seniors may also avoid leaving home for other safety reasons, including fear of injury. ${ }^{24}$ A 2011 survey of Toronto residents also found that the majority of people without AC stay home during hot weather; distance to cooling shelters and lack of transport were frequently cited as barriers to leaving home. ${ }^{25}$ In our survey, however, transportation was not singled out as a main barrier to leaving home (Table 3).

Studies in other areas have reported mixed findings about the relationship between warning awareness and behavior change. ${ }^{13}$ A multicity phone survey found that $90 \%$ of respondents were aware of heat warnings but many did not consider themselves to be vulnerable to heat and few reported changes in their behavior as a result of the 
warnings. ${ }^{26}$ In France, however, increased awareness of heat warnings was positively linked with changes in prevention practices from 2005 to $2006 .{ }^{13}$ Of note, this study took place after the 2003 European heat wave, which killed more than 14,000 people in France, one of the deadliest heat waves ever recorded. ${ }^{27}$ The French public may have been more attuned to the dangers of heat after this devastating event.

The proportion of New Yorkers without AC (11\%) was consistent with estimates from the 2007 NYC Community Health Survey (13\%) and with findings of a multicity phone survey of residents aged $\geq 65$ in Philadelphia, Phoenix, Toronto, and Dayton, Ohio. ${ }^{26,28}$ The finding that many NYC residents own but rarely or never use an AC during hot weather because of costs is also consistent with the multicity survey. ${ }^{26}$ In addition, another multicity study found that densely populated cities—such as New York City—with lower and more variable temperatures during the warm season had more heat-related morbidity/mortality. The prevalence of AC in these cities also tended to be lower than more consistently warmer cities, suggesting that the higher burden of heat mortality in areas with more variable temperatures may be related to home AC prevalence. $^{29}$

Findings from the survey and focus groups suggest that helping the public to understand and evaluate their own risk needs to be a component of heat-health programs, in addition to increasing access to home AC for the most vulnerable and making cooling centers accessible. Promoting "checking in" on vulnerable friends, family members, and neighbors during heat waves could encourage at-risk individuals to use AC and take other protective measures. NYC officials regularly urge the public to check on others during heat emergencies, but the frequency of this behavior had never been assessed. The survey indicated that $54 \%$ of people check on others, a figure that can be used as a baseline to inform efforts to encourage "buddy systems." While encouraging, more work is needed to target and evaluate the efficacy of this strategy for heat illness prevention.

There are several potential limits to our analyses. Closed-ended survey questions may miss information not included as a response option or obscure nuanced and multiple reasons for responses. Respondents may also interpret questions differently. For instance, rather than specifying a temperature to define "very hot weather," respondents were allowed to answer based on their own perceptions of uncomfortable heat, since there is no single "safe" threshold. In addition, response categories for frequency of AC use at home during very hot weather were along a scale of "always," "most of the time," "half the time," "less than half the time" and "never." The "less than half the time" response likely includes levels of AC use that is inadequate to protect health and some levels of use that may be protective. The survey sample size was sufficiently large to estimate prevalences, but was too small to test for statistical differences in prevalence between subgroups in some cases.

No questions were asked about specific health conditions that are known to increase heat susceptibility, such as diabetes or cardiovascular disease. Poor self-rated health has been associated with an increased risk of mortality in many studies, even controlling for covariates such as age and comorbidities, ${ }^{30}$ and the measure has been recognized as a useful indicator for risk assessment. ${ }^{14}$ In NYC, poor-fair self-rated health status has been associated with self-reported chronic health conditions that increase heat susceptibility in the annual NYC Community Health Survey. ${ }^{15}$ However, the relationship between self-rated health and "objective" health status has not been fully described and explained. ${ }^{31}$ A number of factors can affect perceptions, and thus reporting, of one's own health status, such as undiagnosed conditions or cultural norms. Misclassification remains a major limitation to this study, as health status is 
limited to self-reported data. Some heat-vulnerable people likely were not included in the group that we defined as "vulnerable" (using self-rated health status, age, and AC access), while others who are not heat-vulnerable may have been included.

Focus groups provided qualitative information about the knowledge, attitudes, and behaviors of a small group of people, and responses are not meant to be representative samples of seniors and potential caregivers in NYC. Senior participants were relatively mobile and able to attend early evening focus groups. However, seniors in relatively good health today may experience declining health in the future, and can still provide a source of insight into heat-health attitudes and behaviors useful in shaping programs. Finally, a systematic analysis of all heat-related media from the summer of 2011 was not performed.

\section{CONCLUSIONS}

On average, heat waves are the most lethal extreme weather event in the USA. ${ }^{2}$ Despite substantial efforts to prevent heat-related morbidity and mortality, $30 \%$ of the most vulnerable New Yorkers in this study population were unaware of warnings about dangerously hot weather in 2011. Even those who are aware of warnings may not consider themselves to be at risk, or consider AC to be a primary protective strategy during hot weather. Many vulnerable people stay home during hot weather, even if they cannot stay cool there. Other urban areas may face similar challenges.

More effective communication and educational efforts are needed to reach heatvulnerable populations and help motivate effective protective behaviors. More research is needed to understand which risk communications methods and programs are most effective in motivating people to engage in protective behaviors, including use of AC and utilization of cooling centers. In the meantime, heat-health risk communication should emphasize that extreme heat can be deadly, hot indoor environments are dangerous during extreme heat, and that $\mathrm{AC}$ is a life-saving intervention for people who are vulnerable to heat illness. Closer collaboration between public health agencies and meteorologists and others in broadcast media should be explored to help ensure clear and accurate risk communication and amplify the reach and effect of public health messages.

Access to AC was also lower among those with low household income. Increasing access to $\mathrm{AC}$ in the home-for example, assistance with purchasing and operating costs-and enlisting members of the general public to check on heat-vulnerable social contacts, including informing them of effective ways to protect themselves and actively assisting them in staying cool, will also likely be important strategies to avert illness and death during heat waves, now, and in the future.

\section{ACKNOWLEDGMENTS}

We gratefully thank Jesica Rodriguez-Lopez, MPH; Carla Toro, MS, MPH; Sarah Walters, $\mathrm{MPH}$; and the Global Strategy Group, as well as other NYC DOHMH colleagues who have contributed to the project and the CDC's Climate-Ready Cities and States Initiative.

Funding. This work was supported by Centers for Disease Control and Prevention (CDC) grant \#1UE1EH000757-01 and by City of New York tax levy funds.

Open Access This article is distributed under the terms of the Creative Commons Attribution License which permits any use, distribution, and reproduction in any medium, provided the original author(s) and the source are credited. 


\section{REFERENCES}

1. Thacker MT, et al. Overview of deaths associated with natural events, United States, 1979-2004. Disasters. 2008; 32(2): 303-315.

2. National Weather Service. Natural Hazard Statistics. 2013. http://www.nws.noaa.gov/ om/hazstats.shtml. Accessed April 16, 2013

3. O’Neill MS, Ebi KL. Temperature extremes and health: impacts of climate variability and change in the United States. J Occup Environ Med. 2009; 51(1): 13-25.

4. NYC Department of Health and Mental Hygiene. NYC Vital Signs Investigation Report: Deaths associated with heat waves in 2006. http://www.nyc.gov/html/doh/downloads/ pdf/survey/survey-2006heatdeaths.pdf

5. Centers for Disease Control and Prevention. Heat Illness and Deaths - New York City, 2000-2011. MMWR. 2013; 62(31): 617-621.

6. New York City Panel on Climate Change (NPCC). Climate risk information, 2010. New York, NY: NPCC; 2010.

7. Kilbourne EM, et al. Risk factors for heatstroke: a case-control study. JAMA. 1982; 247(24): 3332-3336.

8. Semenza JC, et al. Heat-related deaths during the July 1995 heat wave in Chicago. $N$ Engl J Med. 1996; 335(2): 84-90.

9. Semenza JC, et al. Excess hospital admissions during the July 1995 heat wave in Chicago. Am J Prev Med. 1999; 16(4): 269-277.

10. Anderson GB, Bell ML. Heat waves in the United States: mortality risk during heat waves and effect modification by heat wave characteristics in 43 U.S. communities. Environ Health Perspect. 2011; 119(2): 210-218.

11. O’Neill MS, Zanobetti A, Schwartz J. Disparities by race in heat-related mortality in four US cities: the role of air conditioning prevalence. J Urban Health. 2005; 82(2): 191-197.

12. US Environmental Protection Agency (EPA). Excessive heat events guidebook 2006. New York: US EPA; 2006. http://www.epa.gov/hiri/about/pdf/EHEguide_final.pdf

13. Bassil KL, Cole DC. Effectiveness of public health interventions in reducing morbidity and mortality during heat episodes: a structured review. Int J Environ Res Public Health. 2010; 7(3): 991-1001.

14. Jylha M. What is self-rated health and why does it predict mortality? Towards a unified conceptual model. Soc Sci Med. 2009; 69(3): 307-316.

15. Elfassy, T.Y., S; Immerwahr, S; Eisenhower, D., Self-rated general health in New York City, in Epi Data Brief 2013, New York City Department of Health \& Mental Hygiene. http://www.nyc.gov/html/doh/downloads/pdf/epi/databrief24.pdf

16. NYC Department of Health \& Mental Hygiene. District public health offices: New York City's commitment to healthier neighborhoods, 2013. http://www.nyc.gov/html/doh/ downloads/pdf/dpho/dpho-brochure.pdf

17. The American Association for Public Opinion Research. Standard Definitions: Final Dispositions of Case Codes and Outcome Rates for Surveys. 7th edition. AAPOR. 2011. http://www.aapor.org/AM/Template.cfm?Section=Standard_Definitions2\&Template=/CM/ ContentDisplay.cfm\&ContentID=3156

18. New York City Department of Health and Mental Hygiene. Epiquery: NYC Interactive Health Data System - Census 2010. http://nyc.gov/health/epiquery

19. White-Newsome JL, et al. Climate change and health: indoor heat exposure in vulnerable populations. Environ Res. 2012; 112: 20-27.

20. Gupta, S., et al., Electric fans for reducing adverse health impacts in heatwaves. Cochrane Database Syst Rev, 2012. 7: p. CD009888

21. Hajat S, O'Connor M, Kosatsky T. Health effects of hot weather: from awareness of risk factors to effective health protection. Lancet. 2010; 375(9717): 856-863.

22. Blum LN, Bresolin LB, Williams MA. From the AMA Council on Scientific Affairs. Heatrelated illness during extreme weather emergencies. JAMA. 1998; 279(19): 1514. 
23. Klinenberg E. Heat wave: a social autopsy of disaster in Chicago. Chicago, IL: University of Chicago Press; 2002.

24. Boyd R, Stevens JA. Falls and fear of falling: burden, beliefs and behaviours. Age Ageing. 2009; 38(4): 423-428.

25. Toronto Public Health. Protecting vulnerable people from health impacts of extreme heat, 2011. Toronto, Ontario: Toronto Public Health; 2011. http://www1.toronto.ca/staticfiles/ city_of_toronto/toronto_public_health/healthy_environment/heat_alert/files/pdf/ backgroundfile-39469.pdf

26. Sheridan SC. A survey of public perception and response to heat warnings across four North American cities: an evaluation of municipal effectiveness. Int J Biometeorol. 2007; 52(1): 3-15.

27. Kovats RS, Hajat S. Heat stress and public health: a critical review. Annu Rev Public Health. 2008; 29: 41-55.

28. NYC Department of Health \& Mental Hygiene. New York City Community Health Survey. 2007. http://www.nyc.gov/html/doh/html/data/survey.shtml. Accessed September 25, 2012

29. Medina-Ramon M, Schwartz J. Temperature, temperature extremes, and mortality: a study of acclimatization and effect modification in 50 United States cities. Occup Environ Med. 2007; 64(12): 827-833.

30. DeSalvo KB, et al. Mortality prediction with a single general self-rated health question. A meta-analysis. J Gen Intern Med. 2006; 21(3): 267-275.

31. Huisman M, Deeg DJ. A commentary on Marja Jylha's "What is self-rated health and why does it predict mortality? Towards a unified conceptual model"(69:3, 2009, 307316). Soc Sci Med. 2010; 70(5): 652-654. discussion 655-7. 